\title{
Elastofibroma dorsi (ED): predictive sign of hematological malignancies
}

\begin{abstract}
Background: Elastofibroma dorsi (ED) is a benign soft tissue tumor. Multiple genetic mutations are reported in ED. The same mutations are reported in hematological malignancies. In this manuscript, we investigate the association between ED and other malignancies and document typical radiological findings of ED.
\end{abstract}

Methods: A case-control study was conducted. From 1973 to 2015, Patients with ED were identified as cases (52 patients). Controls (150) were randomly selected among the non-ED-patients who had CT scans at our institution. Due to variable malignancy subtypes, limited sample sizes, and predominance of diffuse large B-cell lymphoma (DLBCL) in our cohort, malignances were pooled into DLBCL, none-cancer, and other cancers categories. The primary endpoints were the odd ratios (ORs) of nonecancer and other cancers using DLBCL as a reference. The second endpoints included ORs of affected systems using hematological malignancies as a reference.

Results: ED patients had a higher risk of having DLBCL than controls (P-value $=0.029$ and 0.001 for non-cancer and other cancers respectively). ED patients had a higher probability of having hematological malignancies than controls $(\mathrm{P}$-value $=0.002$ for other systems' cancer). On CT, ED showed the typical pattern (isodense and interspersed with hypodense fatty areas). In all patients except two, ED had moderate metabolic activity on PET-CT. On MRI, ED was hypo- to iso-intense and interspersed with hyper-intense fatty areas.

Conclusion: ED patients have a higher probability of developing hematological malignancy, especially DLBCL. ED with typical imaging characteristics in patients with a neoplastic process should not be interpreted as a metastatic disease.
Volume 3 Issue 2 - 2019

\author{
Bilal Mujtaba,' Mohamed Elshikh,' William \\ Murphy,' Behrang Amini,' Peter Wei, ${ }^{2}$ Jesse \\ Rayan, ${ }^{3}$ Ahmed Elsayes, ' Jia Sun, ${ }^{4}$ Naveen \\ Garg' \\ 'Department of Diagnostic Radiology, The University of Texas \\ MD Anderson Cancer Center, Houston, Texas, USA \\ 2Department of Diagnostic and Interventional Radiology, The \\ University of Texas Health Science Center at Houston, Houston, \\ Texas, USA \\ ${ }^{3}$ Department of Diagnostic Radiology, Baylor College of \\ Medicine, Houston, Texas, USA \\ ${ }^{4}$ Department of Biostatistics, The University of Texas MD \\ Anderson Cancer Center, Houston, Texas, USA
}

Correspondence: Bilal Mujtaba, Department of Diagnostic Radiology, The University of Texas MD Anderson Cancer Center, 1515 Holcombe Blvd., Unit I473, Houston, TX 77030, USA, Tel 203-508-0074,Email BMujtaba@mdanderson.org

Received: September 20, 2017| Published: April 01, 2019

\section{Introduction}

Elastofibroma dorsi (ED) is a non-encapsulated benign soft tissue tumor that classically affects the periscapular region. Other anatomical locations involvement has been reported such; olecranon process, stomach, axilla, and intestine. ${ }^{1}$ On autopsy, ED is $24.4 \%$ and $11.2 \%$ prevalent in women and men respectively, with higher incidence in older patients. ${ }^{2}$ On CT, ED prevalence is $2 \%{ }^{3}$ ED is bilateral in $10 \%-66 \%$ of cases. ${ }^{1-5}$ Pathologically, ED is composed of extracellular collagen and elastin matrix interspersed with fatty tissue strands and fibroblasts. ${ }^{1}$ Multiple theories are suggested for ED pathogenesis such; reactive elastic tissue hyperproliferation, aging process, and neoplastic process. ${ }^{1}$ Despite, the indolent clinical behavior of elastofibroma, it shows multiple genetic mutations. ${ }^{6-10}$ Nishio et al., ${ }^{6}$ documented that $33 \%$ of elastofibroma patients have genetic mutations. The most common mutations are $\mathrm{q}$ arm gain in $\mathrm{X}$ and 19 chromosomes. ${ }^{6}$ McComb et al., ${ }^{9}$ reported that chromosome 1 $\mathrm{p}$ arm genetic alterations are present in three cases of elastofibroma. ${ }^{9}$ Also, Vanni et al., ${ }^{8}$ described the same chromosome $1 \mathrm{p}$ arm mutation in addition to $\mathrm{q}$ arm of chromosomes 3 and $6 .{ }^{8}$ Moreover, another report documented chromosomelp arm genetic alterations in two patients with elastofibroma. The same study described multiple genetic mutations in the 2 patients, interestingly including chromosomes 3 and $19 \mathrm{q}$ arm. Most of genetic mutations reported in elastofibroma are also present in multiple hematological malignancies like $p$ arm of chromosome 1 in leukemia, ${ }^{11-15} \mathrm{q}$ of chromosome 3,19 and chromosome $\mathrm{X}$ in B-cell lymphoma. ${ }^{16-18}$ Clinical prevalence of elastofibroma in patients with hematological or solid malignancies is not reported before. Considering this pattern of genetic mutations in elastofibroma and hematological malignancies, we hypothesized that patients with elastofibroma may have a higher predisposition to have hematological malignancies. In this manuscript, we investigate the association between ED and other malignancies and document typical radiological findings of ED.

\section{Methods}

\section{Patient data}

The primary objective of this study is to determinate the associations between Elastofibroma Dorsie and other malignancies. A case-control study was conducted using MD Anderson Cancer Center (MDACC) data from 1973 to 2015. Patients diagnosed and/or treated with ED were identified as cases (52 patients). Controls (150 patients) were randomly selected among the non-ED patients who had $\mathrm{CT}$ scans at MDACC. To increase statistical power, a higher ratio (24) of controls to cases was applied in data collection. Institutional review board with consent waiving was obtained to search our institutional data registry for all identified patients. We retrospectively reviewed patients' medical records and collected demographic, clinical, pathological, radiological data. Of ED patients, 17 patients were pathologically proven and 35 patients were detected on imaging studies for other diseases. Of the pathologically proven patients, no data was available for 3 patients, as their biopsies were referred to our institution for pathological confirmation only and they were excluded. $39(79.6 \%)$ patients with ED had a malignant process. Diffuse large B-cell lymphoma (DLBCL) was the most frequent malignant process in ED patients. 132 patients in the control group had a neoplastic disease. The most common malignancies were colon and lung cancer.

\section{Stastical analysis}

The primary objective of this analysis was to correlate ED status with other malignances. Due to large amount of different malignancy 
subtypes, limited sample sizes, and considering predominance of DLBCL in our cohort, malignances were pooled together into three categories: DLBCL, none cancer, and other cancers. The primary endpoints were the odd ratios (ORs) of none cancer and other cancers when we used DLBCL as the reference group. The second endpoints included the ORs of other affected systems when we used hematological malignancies as the reference group. Covariates included age, sex, and ED status ( $0=$ control and $1=$ case). A total of 49 ED patients and 150 controls were analyzed in this report. Summary statistics of other malignances and patient characteristics, such as age and sex, were provided in frequencies, percentages, mean, SD, and range. As the outcome variable had three levels, a multinomial logistic regression was used to correlate other malignances with ED status. The ORs of a basic model containing a single dichotomous covariate (ED status) were obtained. All tests were two-sided and p-values of 0.05 or less were considered statistically significant. Statistical analysis was carried out using SAS version 9.2 (SAS Institute, Cary, NC).

\section{Results}

In ED patients, Periscapular area was the most affected anatomical location (46 patients; 93.9\%) followed by GIT ( 2 patients; 4.1\%). 24 $(49.7 \%)$ patients had bilateral lesions and 14 (28.6\%) patients had right side ED. ED was incidentally detected in $40(81.6 \%)$ patients, most of them were managed in our institution for malignancy. 8 patients complained of slowly growing mass in their back and one patient presented with chronic periscapular pain. Elastofibroma was surgically removed in 9 patients. $8(15.4 \%)$ patients were manual workers.

\section{Association between ED and other malignancies}

Age was significantly different between elastofibroma patients and control group. Mean age for ED patients was 67.27, while 58.73 for the control group (P-value $=0.0003$ ) (Table 1A). Gender was not significantly different between ED patients and control group $(\mathrm{P}$-value $=0.59)($ Table 1B). $10 \mathrm{ED}$ patients and 15 patients from the control group did not have any neoplastic process. 19 malignant diseases affecting 8 systems were found in elastofibroma patients. Hematological malignancies; namely lymphoma were the most common malignant disease in elastofibroma patients followed by genitourinary malignancies. 56 neoplastic diseases were found in the control group affecting 11 systems; 3 were benign and 53 were malignant. Lung and colon cancer were the most common malignancies in the control group followed by prostate cancer. Of note, only $11(7 \%)$ patients out of 150 in control group had hematological malignancies, while $11(22 \%)$ patients out of 49 in the ED group had hematological malignancies. Moreover, only one $(0.01 \%)$ patient in the control group had DLBCL, on the other hand $8(16.3 \%)$ patients in the ED group had DLBCL. Considering that, DLBCL was the most common malignant disease in ED patients. DLBCL was used as a reference group for comparison of ED patients with the control group. ED patients were at a higher risk of having DLBCL than the control group ( $P$-value $=0.029$ and 0.001 in comparison to non-cancer and other cancers respectively) (Table $2 \mathrm{~B})$. When hematological malignancies was used as reference group, ED patients had a significantly higher probability of having hematological malignancies than the control group in comparison to other systems' cancer ( $\mathrm{P}$-value $=0.002$ ) (Table $2 \mathrm{~A})$. On the other hand, the control group was at a higher risk of having other systems' cancers. Genitourinary (GU) cancers had the same frequency like hematological malignancies in ED patients. When GU cancers were used as a reference group for comparison of ED patients with the control group, ED patients were not at a high of having GU cancers (non-significant $\mathrm{P}$-value $=0.39$ and 0.27 , on comparison with non-cancer and other system cancers respectively).

Table I Summary of patients' age and gender in the two groups

Table I(A) Summary of patients' age (3 patients were deceased). The ages in case and control groups were tested by two sample t-test with equal variances $(P$-value $=0.0003)$.

\begin{tabular}{|c|c|c|c|c|c|c|c|}
\hline & Group & $\mathbf{N}$ & Mean & SD & Min & Median & Max \\
\hline \multirow{3}{*}{ Age } & $\begin{array}{l}\text { Control } \\
\text { (none-ED) }\end{array}$ & 147 & 58.73 & 14.62 & 10 & 61 & 93 \\
\hline & Case (ED) & 49 & 67.27 & 12.06 & 29 & 66 & 87 \\
\hline & All & 196 & 60.87 & 14.48 & 10 & 62.5 & 93 \\
\hline
\end{tabular}

Conclusion: The ages in case and control groups are significant different.

Table I(B) Summary of patient sex. A chi-squared test showed there was no significant difference of sex distribution in case and control groups (P-value $=0.59)$.

\begin{tabular}{lllllll}
\hline \multicolumn{3}{c}{ Group } & \multicolumn{3}{c}{ All } \\
\hline Control (none-ED) & \multicolumn{2}{l}{ Case (ED) } \\
\hline Gender & N & $\%$ & N & $\%$ & N & $\%$ \\
\hline Female & 76 & 73.79 & 27 & 26.21 & 103 & 51.76 \\
Male & 74 & 77.08 & 22 & 22.92 & 96 & 48.24 \\
\hline
\end{tabular}

\section{Imaging findings}

Computed tomography (CT), positron emission tomography (PET)-CT, magnetic resonance imaging (MRI), and ultrasound were used in $37,27,14$, and 2 patients respectively to diagnose and follow up patients with elastofibroma. The average diameter of elastofibroma in our population was $5.8 \mathrm{~cm}$ in the anteroposterior diameter. Follow up imaging was available in 30 patients for 75.5 months on average (range; 10-168 months). 3 of them had surgical removal and no recurrence was detected. Elastofibroma size did not change in 22 patients. On the other hand, it increased in size in 5 patients with a mean increase of $1.7 \mathrm{~cm}$ (Figure 1) with no aggressive features to suggest malignant transformation. In one patient elastofibroma showed marked diameter decrease over 5 months without any treatment, which may be suggestive of a resolving inflammatory process. On $\mathrm{CT}$, ED appeared as an isodense lesion to the muscle interspersed with hypodense areas representing adipose tissue (Figure 1) of our population, 27 patients had PET-CT. In 25 patients, ED showed intermediate FGD avidity, while ED was non-avid in 2 patients. Of those 25 patients, elasstofibroma became non FDG-avid in one patient after 3 years of follow up; this finding was associated with evident fatty degeneration on CT. On MRI, elastofibroma was iso to hypo-intense to the muscle with interspersed hyper-intense fatty areas (Figure 2) in all patients except one. In this patient, ED was described to be hyperintense on T2 series suggesting of an inflammatory process. With contrast enhanced series, all elastofibroma showed heterogeneous pattern of enhancement. On ultrasonography, elastofibroma appeared as hypoechoic vascular mass in one patient and heterogeneous soft tissue echogenic mass in another one.

\section{Discussion}

To our knowledge, the association of ED with other malignancies was not reported in the literature before. We conducted this study to examine the association of ED with other malignancies. We found that ED patients are at a statistically significant higher risk of developing 
hematological malignancies (P-value $=0.002)$, specifically DLBCL compared to the control group. This finding may be explained by the shared genetic mutations between ED and hematological malignancies. Chromosomal instability and multiple genetic breakpoints were the salient cytogenetic feature of ED..$^{7-10}$ The most common reported genetic mutations in ED were chromosome $1 \mathrm{p}$ arm, chromosomes 3, 19 and $\mathrm{X} \mathrm{q}$ arms. ${ }^{7-10}$ Chromosome $1 \mathrm{p} 32$ breakpoint was frequently reported in ED patients. ${ }^{8,9}$ Multiple translocations involving $1 \mathrm{p} 32$ genetic location is reported in several types of leukemia (acute T, B, megakaryocytic, lymphoblastic and monocytic leukemia). ${ }^{11-15}$ In our population, DLBCL was the most common malignancy in patients with ED and its incidence was statistically higher in ED patients than the control group in comparison to other cancers (P-value $=0.001)$. Bea et al documented multiple mutations of $3 q$ arm in $41 \%$ of activated B-cell-like DLBCL (26\% gain and 15\% trisomy). They also reported gain mutation of $\mathrm{X}$ chromosome in $13 \%$ of DLBCL patients. ${ }^{21}$ In another series, gain mutation in chromosome 3 (q23-q28) was detected in $25 \%$ to $36 \%$ of activated B-cell-like DLBCL. ${ }^{16}$ In three reports, structural abnormalities at the $\mathrm{q}$ arm of chromosome 3 in $6 \mathrm{ED}$ in 5 patients were documented. ${ }^{7-10}$ In another report, the most common genetic mutation in elastofibroma patients (6 patients, $22 \%$ ) was a gain in $\mathrm{q}$ arm of the $\mathrm{X}$ chromosome. ${ }^{6}$ Additionally, Hammond et al documented a $32 \%$ and $24 \%$ incidence of gain mutations in $\mathrm{X}$ and 3 chromosomes respectively in Non-Hodgkin lymphoma (NHL) patients. ${ }^{17}$ Interestingly, DLBCL is the most common subtype of NHL. All (11) but one lymphoma patients who had elastofibroma in our population are NHL ( 8 DLBCL and 2 follicular lymphoma). The aforementioned genetic mutations patterns in ED patients and other hematological malignancies might explain the significant higher risk of hematological malignancies in ED patients in our cohort. However, more studies involving cytogenetic analysis are required to add more insights and consolidate this finding.

Table 2 Summary of multinomial logistic regression models.

Table 2(A) Summary of multinomial logistic regression model comparing the probabilities of having different affected systems when use hematology as the reference group. An odds ratio more than I means a higher probability of that affected system among the controls than among the cases. For example, patients in the control group (none-ED) had significantly higher probability of with other affected systems compared with patients in the case group (ED) (OR=4.4,95\% Cl: I.7-I I.2, P-value $=0.002$ )

\begin{tabular}{llllll}
\hline Affected system & Group & Odds Ratio (OR) & $\mathbf{9 5 \%}$ Confidence interval of OR & P-value \\
\hline None cancer vs. hematology & control vs. case & 1.5 & 0.5 & 4.8 & 0.492 \\
$\begin{array}{l}\text { Other systems vs. hematology } \\
\text { The above table is equivalent to below: }\end{array}$ & control vs. case & 4.4 & 1.7 & 11.2 & 0.002 \\
$\begin{array}{l}\text { Hematology vs. none cancer } \\
\text { Hematology vs. other systems }\end{array}$ & case vs. control & 1.5 & 0.5 & 4.8 & 0.492 \\
\hline
\end{tabular}

Conclusion: I. ED patients showed higher probability of having hematological malignancies than none-ED patients when use other systems as the reference group.

Table 2(B) Summary of multinomial logistic regression model comparing the probabilities of having different cancer subtypes when uses diffuse large B cell lymphoma as the reference group.An odds ratio more than I means a higher probability of that malignancy category among the controls than among the cases. For example, patients in the control group (none-ED) had significantly higher probability of other types of cancer compared with patients in the case group (ED) $(\mathrm{OR}=34.6,95 \% \mathrm{Cl}: 4.2-286.7, \mathrm{P}$-value $=0.00 \mathrm{I})$. If we switch the directions in both cancer subtype and group, the ORs will not change. In other words, the odds among ED patients had the disease of diffuse large B cell lymphoma is 34.6 times greater than the odds among none-ED patients when use other cancers as the reference group. The confidence interval indicates that the odds could be as a little as 4.2 times or as much as 286.7 times larger with 95 percent confidence.

\begin{tabular}{|c|c|c|c|c|c|}
\hline \multirow{2}{*}{$\begin{array}{l}\text { Cancer subtype } \\
\text { None cancer vs. large B cell lymphoma }\end{array}$} & \multirow{2}{*}{$\begin{array}{l}\text { Group } \\
\text { control vs. case }\end{array}$} & \multirow{2}{*}{$\begin{array}{l}\text { OR } \\
12\end{array}$} & \multicolumn{2}{|c|}{$95 \%$ Confidence interval of OR } & \multirow{2}{*}{$\begin{array}{l}\text { P-value } \\
0.029\end{array}$} \\
\hline & & & 1.3 & 111.3 & \\
\hline Other cancers vs. large B cell lymphoma & control vs. case & 34.6 & 4.2 & 286.7 & 0.001 \\
\hline \multicolumn{6}{|l|}{ The above table is equivalent to below: } \\
\hline large B cell lymphoma vs. none cancer & case vs. control & 12 & 1.3 & 111.3 & 0.029 \\
\hline large B cell lymphoma vs. other cancers & case vs. control & 34.6 & 4.2 & 286.7 & 0.001 \\
\hline
\end{tabular}

Conclusion: ED patients showed higher probability had diffuse large B cell lymphoma than none-ED patients when use other cancers as the reference group. ED disease is a significant factor in having the disease of large B cell lymphoma. ED patients showed higher probability had diffuse large B cell lymphoma than none-ED patients when use none cancer as the reference group.

Elastofibroma dorsi is a benign soft tissue lesion that affects classically the periscapular area. Many theories have been proposed for ED pathogenesis. Reactive elastic tissue hyperprofileration due to repetitive micro-trauma and friction between chest wall and scapular tip was accepted to be the initiative event for elastofibromatous changes in the periscapular area. ${ }^{19}$ Even though, multiple reports described elastofibroma and pre-elastofibroma changes in nonmanual workers. El Hammoumi et al., ${ }^{1}$ documented history of repetitive occupational trauma in only $25 \%$ of their population. From our cohort, only 8 patients were manual workers representing $15.4 \%$ of ED patients. Elastofibroma is more common in elderly patients.
Some authors considered it as an aging process. In an autopsy study, elastofibroma and elastofibroma like changes were found to be very rare at age less 66 year old. ${ }^{19}$ In this study, age was found to be statistically different between ED patients and the control group (P-value $=0.0003$ ). The mean age of ED patients was 67.27 year and $71.4 \%$ of our cohort were older than 60 year old. Other reports argued that elastofibroma is a neoplastic process due to detected genetic mutations. ${ }^{7-10}$ On imaging, ED classically appears as variablydefined, soft tissue mass in the periscapular area. On CT, ED has tissue density similar to the surrounding skeletal muscles interrupted with hypodense streaks of fatty tissue..$^{5-24}$ However, ED may be 
isodense to the muscle without any hypodense areas. ${ }^{3-24}$ In this study, all ED showed the typical appearance on CT, but hypodense fatty areas variates from a lesion to another. As on CT, ED on MRI has signal intensity similar to the muscle interspersed with hyperintense adipose tissue on both $\mathrm{T} 1$ and $\mathrm{T} 2$ series. $^{5-25}$ On contrast, ED has a heterogeneous pattern of enhancement. ${ }^{5-25}$ All patients in this cohort showed the typical pattern of elastofibroma on MRI except in one patient, the lesion was described to be hyperintense on T2 weighted images. On contrast enhanced MRI, all lesions showed heterogeneous enhancement. On PET-CT, elastofibroma usually shows mild to moderate FDG avidity. ${ }^{26-32}$ However, $5.3 \%$ and $23 \%$ of elastofibroma were described to be non-FDG avid in two reports. ${ }^{26,27}$ In this study $92.6 \%$ of elastofibroma showed intermediate FDG avidity, and it was non-avid in $7.4 \%$ of patients. In one patient, elastofibroma became non-FDG avid after 3 years of follow up and this was associated with fatty degeneration on CT. On follow up imaging, Erhamamci et al., ${ }^{32}$ reported no change in elastofibroma size or metabolic activity after chemotherapy. They suggested this finding as an evidence of the benign nature of ED. ${ }^{32}$ Wasyliw et al., ${ }^{29}$ also documented no size variation in ED over 2 years of follow up. In this series, follow up imaging was available in 30 patients over 75.5 month on average. No recurrence was detected for surgically removed lesions. ED remained stable in $78.6 \%$ of patients, increased in size in $17.9 \%$ of patients with no aggressive features documented. In one patient, the lesion decreased in size over 5 months, which may be explained by accompanied inflammatory process. Limitations of our study were retrospective nature, not all patients were pathologically proven, but the pattern on imaging studies was specific, and no genetic testing was available.

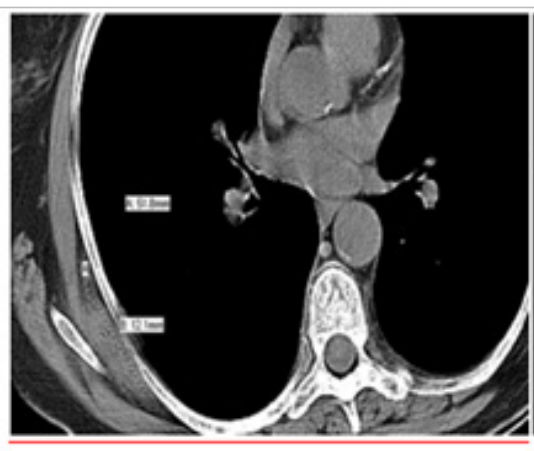

A

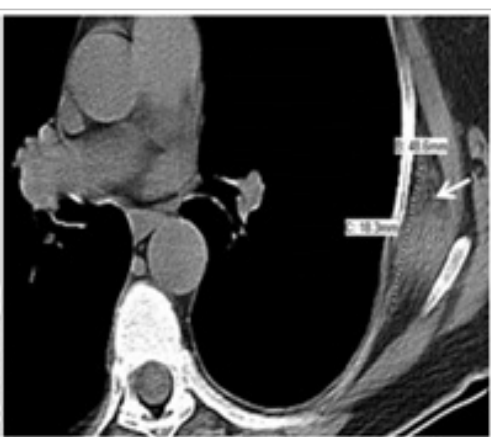

B

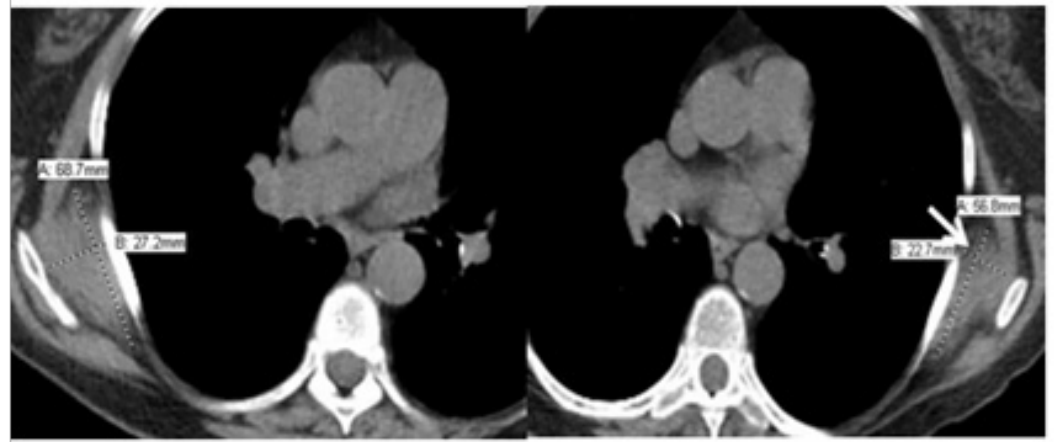

$\mathrm{C}$

D

Figure I CT of bilateral elastofibroma in an 86-year-old Caucasian female who had large B-cell lymphoma. Non contrast enhance axial CT of chest showed (a, b) isodense bilateral subscapular mass with hypodense fatty areas (arrows). The lesion increased in size over 10 years of follow up (c, d) without any aggressive features.

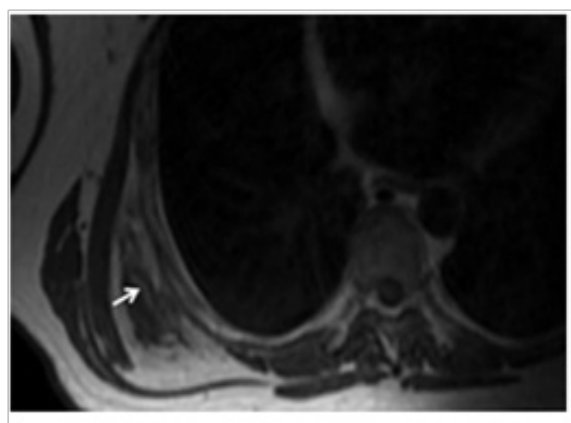

A

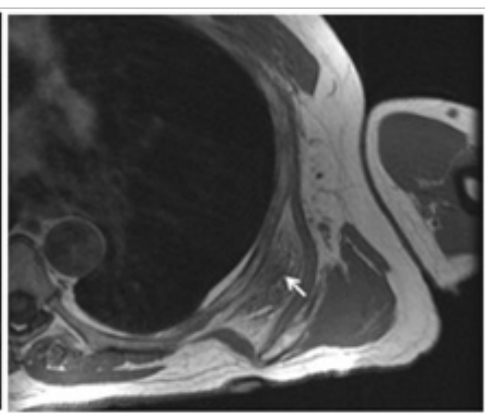

B

Figure 2 Chest MRI. For patient I, a 47-year-old Caucasian man who presented with mass sensation in his back. TI weighted MRI (a) showed a right side isointense subscapular mass interspersed with hyperintense areas (arrow). This mass was proven to be elastofibroma pathologically. For patient 2 , a 75 year old Hispanic man who also complained of slowly growing mass in his left back which had (B) a soft tissue density isointense to the muscle and interrupted with hyperintense areas (arrow). 


\section{Conclusion}

ED patients are at a higher risk of developing hematological malignancies; especially diffuse large B-cell lymphoma. This association is likely secondary to the shared chromosomal mutations between elastofibroma and hematological malignancies ( $p$ arm of chromosome 1 and $\mathrm{q}$ arm of chromosomes 3 and X). Genetic testing is recommended for patient with $\mathrm{ED}$, and clinical surveillance is warranted for patients with the aforementioned mutations. ED presenting with the typical imaging characteristics in a patient with a neoplastic process should not be interpreted as a metastatic disease.

\section{Acknowledgments}

None.

\section{Conflicts of interest}

The author declares there is no conflict of interest.

\section{References}

1. El Hammoumi M, Qtaibi A, Arsalane A, et al. Elastofibroma dorsi: clinicopathological analysis of 76 cases. Korean J Thorac Cardiovasc Surg. 2014;47(2):111-116.

2. Jarvi $\mathrm{OH}, \mathrm{PH}$ Lansimies. Subclinical elastofibromas in the scapular region in an autopsy series. Acta Pathol Microbiol Scand A. 1975;83(1):87-108.

3. Brandser EA, JC Goree, GY El-Khoury. Elastofibroma dorsi: prevalence in an elderly patient population as revealed by CT. AJR Am J Roentgenol. 1998;171(4):977-780.

4. Di Vito A, E Scali, G Ferraro, et al. Elastofibroma dorsi: a histochemical and immunohistochemical study. Eur J Histochem. 2015;59(1):2459.

5. Kransdorf M, J Meis, E Montgomery. Elastofibroma: MR and CT appearance with radiologic-pathologic correlation. AJR Am J Roentgenol. 1992;159(3):575-579.

6. Nishio J, H Iwasaki, Y Ohjimi, et al. Gain of Xq detected by comparative genomic hybridization in elastofibroma. International journal of molecular medicine. 2002;10(3):277-280.

7. Hernández JLG, JO Rodríguez-Parets, JM Valero, et al. High-resolution genome-wide analysis of chromosomal alterations in elastofibroma. Virchows Archiv. 2010;456(6):681-687.

8. Vanni R, S Marras, G Faa, et al. Chromosome instability in elastofibroma. Cancer Genet Cytogenet. 1999;111(2):182-183.

9. McComb EN, MG Feely, JR Neff, et al. Cytogenetic instability, predominantly involving chromosome 1, is characteristic of elastofibroma. Cancer Genet Cytogenet. 2001;126(1):68-72.

10. Batstone P, L Forsyth, J Goodlad. Clonal chromosome aberrations secondary to chromosome instability in an elastofibroma. Cancer Genet Cytogenet. 2001;128(1):46-47.

11. Kotecha RS, A Murch, U Kees, et al. Pre-natal, clonal origin of $\mathrm{t}(1 ; 11)$ (p32;q23) acute lymphoblastic leukemia in monozygotic twins. Leuk Res. 2012;36(1):46-50.

12. Bernard O, C Barin, C. Charrin, et al. Characterization of translocation $\mathrm{t}(1 ; 14)(\mathrm{p} 32 ; \mathrm{q} 11)$ in a $\mathrm{T}$ and in a $\mathrm{B}$ acute leukemia. Leukemia. 1993;7(10):1509-1513.

13. Sagawa M, T Shimizu, T Shimizu. Establishment of a new human acute monocytic leukemia cell line TZ-1 with $\mathrm{t}(1 ; 11)(\mathrm{p} 32 ; \mathrm{q} 23)$ and fusion gene MLL-EPS15. Leukemia. 2006;20(9):1566-1571.
14. Fitzgerald TJ, GA Neale, SC Raimondi, et al. c-tal, a helix-loophelix protein, is juxtaposed to the T-cell receptor-beta chain gene by a reciprocal chromosomal translocation: $\mathrm{t}(1 ; 7)(\mathrm{p} 32 ; \mathrm{q} 35)$. Blood. 1991;78(10):2686-2695.

15. Hasegawa D, K Sano, Y Kosaka, et al. Acute megakaryoblastic leukemia in an infant with a novel $\mathrm{t}(1 ; 9)(\mathrm{p} 32 ; \mathrm{q} 34)$. Cancer Genet Cytogenet. 2000;122(1):59-62.

16. Tagawa H, M Suguro, S Tsuzuki, et al. Comparison of genome profiles for identification of distinct subgroups of diffuse large B-cell lymphoma. Blood. 2005;106(5):1770-1777.

17. Hammond DW, JR Goepel, M Aitken, et al. Cytogenetic analysis of a United Kingdom series of non-Hodgkins lymphomas. Cancer Genet Cytogenet. 1992;61(1):31-38.

18. Cigudosa JC, NZ Parsa, DC Louie, et al. Cytogenetic analysis of 363 consecutively ascertained diffuse large B-cell lymphomas. Genes Chromosomes Cancer. 1999;25(2):123-133.

19. Giebel G, E Bierhoff, J Vogel. Elastofibroma and pre-elastofibroma-a biopsy and autopsy study. European Journal of Surgical Oncology (EJSO). 1996;22(1):93-96.

20. Akcam TI, U Cagirici, A Cakan, et al. Bilateral familial elastofibroma dorsi: is genetic abnormality essential? Ann Thorac Surg. 2014;98(2):e31-e32.

21. Bea S, A Zettl, G Wright, et al. Diffuse large B-cell lymphoma subgroups have distinct genetic profiles that influence tumor biology and improve gene-expression-based survival prediction. Blood. 2005;106(9):3183-3190.

22. Soler R, I Requejo, F Pombo, et al. Elastofibroma dorsi: MR and CT findings. European journal of radiology. 1998;27(3):264-267.

23. Ochsner JE, SA Sewall, GN Brooks, et al. Elastofibroma Dorsi 1. Radiographics. 2006;26(6):1873-1876.

24. Naylor M, A Nascimento, A Sherrick, et al. Elastofibroma dorsi: radiologic findings in 12 patients. AJR American journal of roentgenology. 1996;167(3):683-687.

25. Muratori F, M Esposito, F Rosa, et al. Elastofibroma dorsi: 8 case reports and a literature review. J Orthop Traumatol. 2008;9(1):33-37.

26. Onishi Y, K Kitajima, M Senda, et al. FDG-PET/CT imaging of elastofibroma dorsi. Skeletal radiol. 2011;40(7):849-853.

27. Fang N, Yl Wang, L Zeng, et al. Characteristics of elastofibroma dors on PET/CT imaging with 18 F-FDG. Clinical imaging. 2016;40(1):110 113.

28. Patrikeos A, W Breidahl, P Robins. F-18 FDG uptake associated with elastofibroma dorsi. Clin Nucl Med. 2005;30(9):617-618.

29. Wasyliw CW, VJ Caride. Incidental detection of bilateral elastofibroma dorsi with F-18 FDG PET/CT. Clin Nucl Med. 2005;30(10):700-701.

30. Pierce III JC, R Henderson. Hypermetabolism of elastofibroma dorsi on PET-CT. AJR Am J Roentgenol. 2004;183(1):35-37.

31. Blumenkrantz Y, GL Bruno, CJ González, et al. Characterization of elastofibroma dorsi with 18 FDG PET/CT: a retrospective study. Revista española de medicina nuclear. 2011;30(6):342-345.

32. Erhamamci S, M Reyhan, GN Nursal, et al. Elastofibroma dorsi incidentally detected by $18 \mathrm{~F}-\mathrm{FDG}$ PET/CT imaging. Ann Nucl Med. 2015;29(5):420-425. 